INGENIERÍA ELÉCTRICA Y ELECTRÓNICA

\title{
Criterios de diseño para un túnel de agua de bajo costo
}

ELECTRIC AND ELECTRONICS ENGINEERING

\section{Design criteria for a low cost water tunnel}

\author{
Germán A. Ibarra*, Jairo A. Palacios** \\ Grupo de Investigación en Conversión de Energía (CONVERGÍA), \\ Escuela de Ingeniería Eléctrica y Electrónica, Universidad del Valle. \\ §german.ibarra@correounivalle.edu.co,jairo.palacios@correounivalle.edu.co
}

(Recibido: Junio 4 de 2012 - Aceptado: Abril 16 de 2013 )

\section{Resumen}

Se presentan algunos criterios de diseño para un Túnel de agua, un tipo de instalación orientada a la investigación experimental en áreas relacionadas con la Mecánica de Fluidos. Se proponen estrategias de ahorro energético y un diseño compacto para favorecer su viabilidad en países como Colombia donde no son muy comunes. La clasificación del circuito de flujo se hace en dos zonas, la Zona principal y el sistema de bombeo, la cual determinará la configuración del acoplamiento mutuo para cerrar el circuito. Una metodología de diseño es propuesta y aplicada para el diseño de un túnel orientado hacia la evaluación experimental de nuevas Máquinas Hidrocinéticas (MH) y Pequeños Submarinos (PS), ambos utilizados en el campo de la generación de energía renovable en pequeña escala a partir de recursos hídricos

Palabras clave: Túneles de agua, pruebas experimentales para energía renovable, pequeños submarinos, máquinas hidrocinéticas, generación de energía.

\begin{abstract}
Some criteria are presented for the design of a Water tunnel, a kind of facility for experimental research related with Fluid Mechanics. Energy saving strategies and a compact configuration are proposed for increase their feasibility in countries like Colombia where they are not known in detail. Elements in the closed loop are classified in two zones, Main Zone and Pumping System; the latter will condition the configuration to make the coupling and close the circuit. It is proposed a design methodology and applied for a water tunnel design to be used in experimental tests for new Hydrokinetic Machines (HM) and Small Underwater Vehicles (SUV), both used in energy generation in small-scale renewable energy from water resources.
\end{abstract}

Keywords: Water tunnels, test facilities for renewable energy, small underwater vehicles, hydrokinetic machines. 


\section{Introducción}

En las áreas relacionadas con la mecánica de fluidos y la transferencia de calor y masa, la experimentación es la fuente primaria de informaciónylabase para otorgarvalidezamodelos teóricos y computacionales. Así se investigan en la actualidad numerosos fenómenos aunque sus resultados dependen de la instrumentación e instalaciones disponibles. Los túneles de agua, geométricamente similares a los más conocidos túneles de viento, son instalaciones de pruebas experimentales generalmente en circuito cerrado donde se bombea agua en diferentes condiciones para estudiar su interacción con el objeto bajo estudio en una zona de prueba. El objeto puede tener algún tipo de superficie lisa o rugosa, térmicamente estable o inestable, en reposo o en movimiento de traslación y/o rotación, inerte o incluso algún tipo de ser vivo.
Los túneles de viento y de agua han sido parte fundamental del desarrollo de diversas ramas del conocimiento. Como evidencia de esto están los primeros estudios sobre Fuerzas aerodinámicas sobre perfiles aerodinámicos en 1901, Capa Límite en 1904, Convección Forzada en 1917, entre otros (Shaughnessy et al, 2005) (Lienhard IV \& Lienhard, V, 2003). Los estudios modernos se amplían hacia temas como cavitación ( $\mathrm{LMH}$, 2011), diseño de turbomáquinas y aeronaves (ASME, 2011) (Cummings \& Morton, 2006), flujo interno a través de varios componentes (Klemm \& Gabi, 2003), evaluación de prototipos (Bahaj et al, 2007), efectos de la separación de flujo sobre superficies y cuerpos (Subramanian et al, 2004) (VHE, 2012), sistemas de propulsión (Germain, 2006), desarrollo de órganos artificiales (Lin et al, 2006), captura o almacenamiento de $\mathrm{CO} 2$ (Warzinski, 2000), estudio de la hidrodinámica
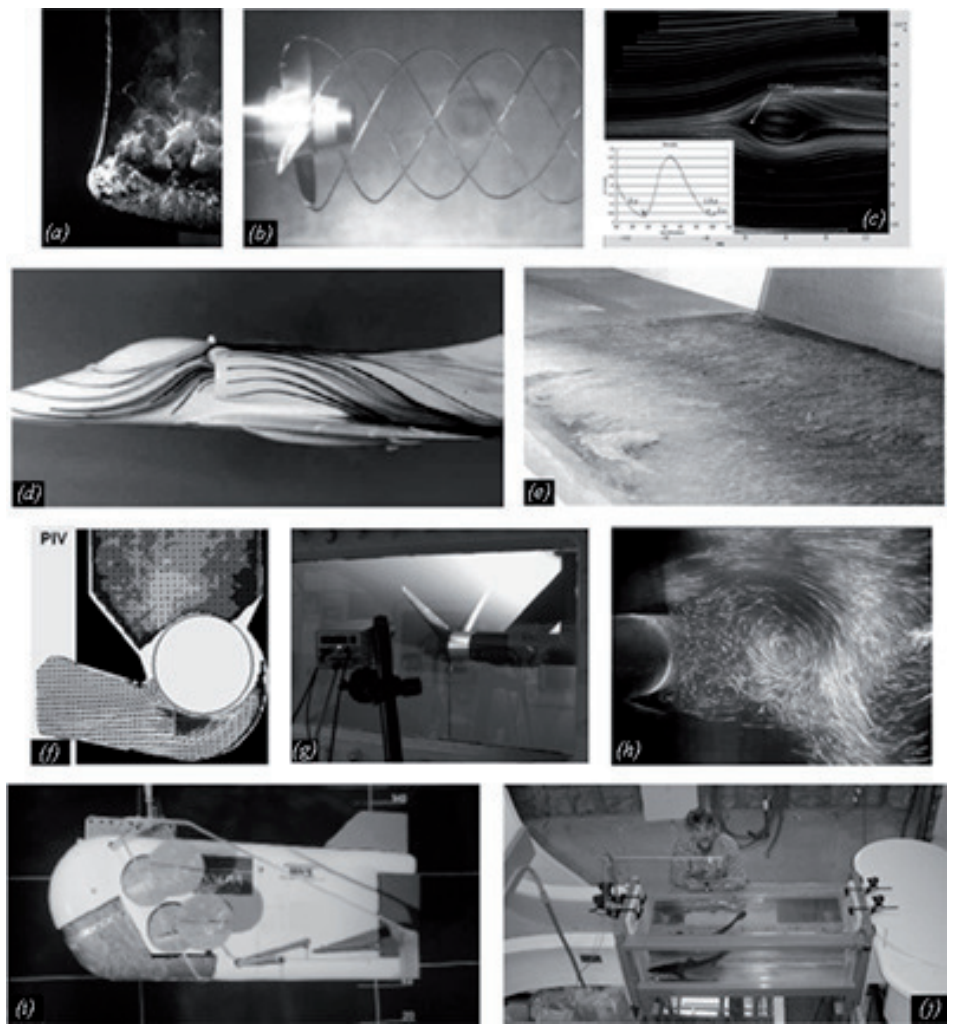

Figura 1. Algunas investigaciones en túneles de agua. (a) Cavitación sobre un perfil NACA 16020, (b) cavitación en propulsión, (c) líneas de corriente: flujo pulsátil sobre un cilindro para el desarrollo de un pulmón artificial, (d) líneas de corriente alrededor de un avión supersónico, (e) estudio de la capa límite en superficies cubiertas por algas, (f) validación de modelos computacionales con técnicas acústicas en un ventilador centrífugo, $(g)$ y $(h)$ generación eléctrica con nuevas máquinas hidráulicas, (h) pequeño submarino, (i) estudio de la locomoción de tiburones. Ver el numeral 7.1 las referencias asociadas. 
y locomoción de animales subacuáticos (FML, 2012), entre otros (Figura.1).

En estos experimentos el flujo incidente debe tener lo que se conoce como Perfil de velocidades uniforme, que se confunde comúnmente con el orden propio del Flujo Laminar. Esa uniformidad se consigue en flujo turbulento antes de que se obtenga un perfil completamente desarrollado en el interior del conducto, esto es, con un pequeño espesor de la Capa límite del flujo viscoso en contacto con las fronteras sólidas. Así, el gradiente de velocidades que se genera en dirección perpendicular al flujo depende en gran parte del Número de Reynolds y tiende a ser plano aún con flujo turbulento, de hecho, esta condición de frontera es la que se suele utilizar en la Dinámica de Fluidos Computacional. La necesidad de generar un perfil de velocidades uniforme surge porque se busca analizar el comportamiento del flujo debido solamente a la interacción con el objeto de estudio.

Este trabajo se basa en una investigación acerca de las estrategias que pueden ser consideradas en el diseño y construcción de un túnel de agua de mediano tamaño, en este caso, para la evaluación experimental de nuevas máquinas hidrocinéticas (MH) y pequeños submarinos (PS) utilizados en el campo de la generación de energía en pequeña escala.

\section{Elementos para el diseño}

El diseño de estas instalaciones comprende dos partes: una estructural y otra hidrodinámica. La primera se adapta a los requerimientos dictados por la hidrodinámica $\mathrm{y}$ debe apoyarse en la aplicación de normas para recipientes a presión según el tamaño de la instalación y la normativa de cada región (el código ASME es uno de los más reconocidos en el mundo).

En el diseño hidrodinámico, la Zona de pruebas (Test section) es la más importante y sus características determinarán en gran parte el tamaño y el consumo energético de toda la instalación. Esas características dependerán del tamaño de los modelos bajo estudio que se rige por las Leyes de Similitud y el Análisis dimensional. Cuando la relación entre las áreas transversales entre modelo y Zona de prueba es cercana a 1.0 las fronteras sólidas condicionan el comportamiento del flujo, por lo cual se deben calcular los factores de corrección adecuados para las variables medidas dependiendo del tipo de geometría y movimiento del modelo.

Como resultado, las características de la Zona de prueba más importantes son: área transversal (forma y tamaño), longitud, intensidad de turbulencia $(<3 \%)$, rango de velocidades, temperatura y parámetros adimensionales (Números de Reynolds, Froude, Webber, Strouhal, etc.). La versatilidad debe ser una cualidad valiosa para obtener un túnel con buena relación costo/ beneficio, teniendo en cuenta los costos por la cantidad de agua necesaria, el sistema de bombeo, los materiales de construcción y la fabricación.

Previa a la Zona de prueba, seinstalan generalmente dos secciones juntas para obtener más fácilmente el perfil de velocidades adecuado: la primera, donde se elimina gran parte de la turbulencia (Settling zone) y la segunda, corresponde a un cambio de sección que une ésta última para que converja y descargue el agua en la Zona de prueba (Contraction section) a la velocidad requerida por el experimento. Ambas secciones suelen ser de igual y gran área transversal, entre $4-9$ veces el área transversal de la Zona de pruebas, lo que implica una gran cantidad de agua. El acondicionamiento del flujo se realiza en la primera sección, donde se elimina gran parte de las fluctuaciones transversales y longitudinales de la velocidad por medio de una serie de pantallas especiales formadas por celdas (circulares, hexagonales o rectangulares) y mallas de alambre entrelazado, respectivamente. Todos los túneles convencionales poseen esta primera sección, no obstante, algunos no tienen contracción o se reemplaza por un difusor que descarga el agua hacia la Zona de prueba; ver Figura 2.

Una vez el fluido interacciona con el modelo, su comportamiento se torna irregular favoreciendo 


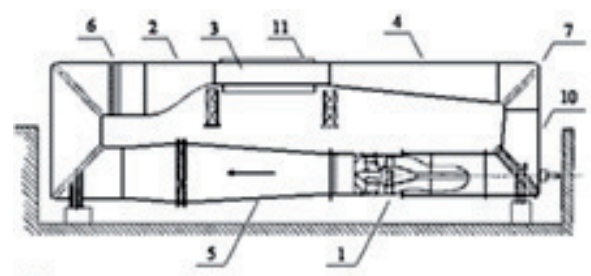

(a)

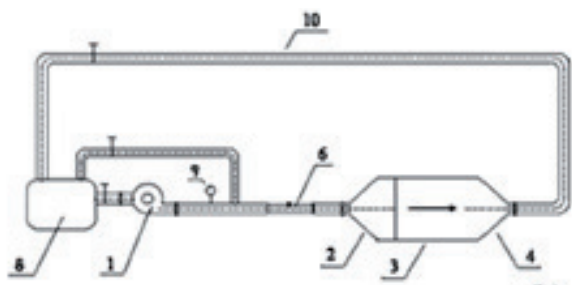

(b)

(c)
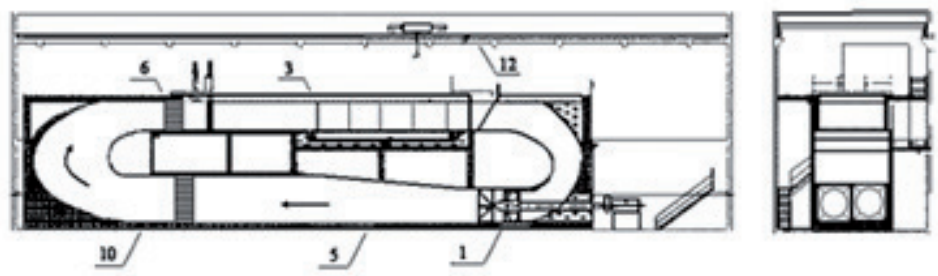

Figura 2. Tipos de túneles de agua clasificados de acuerdo a los elementos previos a la Zona de prueba. (a) Con contracción (convencionales), (b) con difusor y (c).sección transversal constante. Nomenclatura: 1-Bomba, 2-Contracción, 3-Zona de prueba, 4-Difusor, 5-Cambio de sección/difusor, 6-Pantallas, 7-Codos especiales, 8-Tanque de succión, 9-Medidor de flujo, 10-Tubería/conducto, 11-Cámara acústica (opcional), 12-Puente grúa y diferencial (opcional).

las pérdidas de energía debidas a su vorticidad. Para disminuir estos efectos se suele instalar un cambio de sección divergente a la salida de la Zona de prueba para disminuir la velocidad del fluido y así pueda regresar a la bomba adecuadamente para ser impulsada nuevamente. Según el caso, los cambios de dirección (o codos) pueden variar en número, sección transversal y posición; además, deben disponer de elementos que ayuden a desviar el flujo disminuyendo las pérdidas en el cambio de dirección. La geometría de estos elementos, generalmente álabes o partes curveadas, debe ser respetada fielmente en el proceso de construcción para una generación mínima de zonas de recirculación. La elección del material de construcción determinará el proceso de manufactura adecuado: los materiales comunes son: el acero (al carbono e inoxidable) y los materiales compuestos. Para profundizar más en el tema se sugiere consultar las recomendaciones en Ibarra (2010), Gleed \& Saiva (1967) y Ripken (1951).

\section{Metodología}

El análisis de la información presentada en referencias como Gleed \& Saiva (1967) y Ripken (1951), ha permitido proponer una secuencia de pasos para el diseño de algunos túneles de agua convencionales en el caso de Ibarra (2010), aquellos que cuentan con una contracción previa a la Zona de pruebas. En esta investigación se extiende el alcance y se propone una metodología para el diseño de instalaciones con difusor previo pensando en la reducción en la cantidad de agua, costos de construcción y mantenimiento, y materiales disponibles en el mercado nacional. La metodología, ilustrada en la Figura 3, tiene tres pasos de diseño para: La zona principal (zona de prueba y elementos inmediatamente anteriores y posteriores), el sistema de bombeo (tubería, accesorios), y por último, las fases generales de la construcción.

\section{Resultados del proceso de diseño}

Para la aplicación considerada en este caso, los puntos en la metodología se desarrollan a continuación siguiendo algunas recomendaciones: Shanghai Jiao Tong University, China (Yang, B. 2012).

\subsection{Zona principal del túnel}

4.1.1 Tipo de estudio: Las pruebas de MH para ríos y PS se realizan con agua limpia a temperatura ambiente, sin generación de cavitación, flujo interno y con superficie libre; existen otro tipo de 


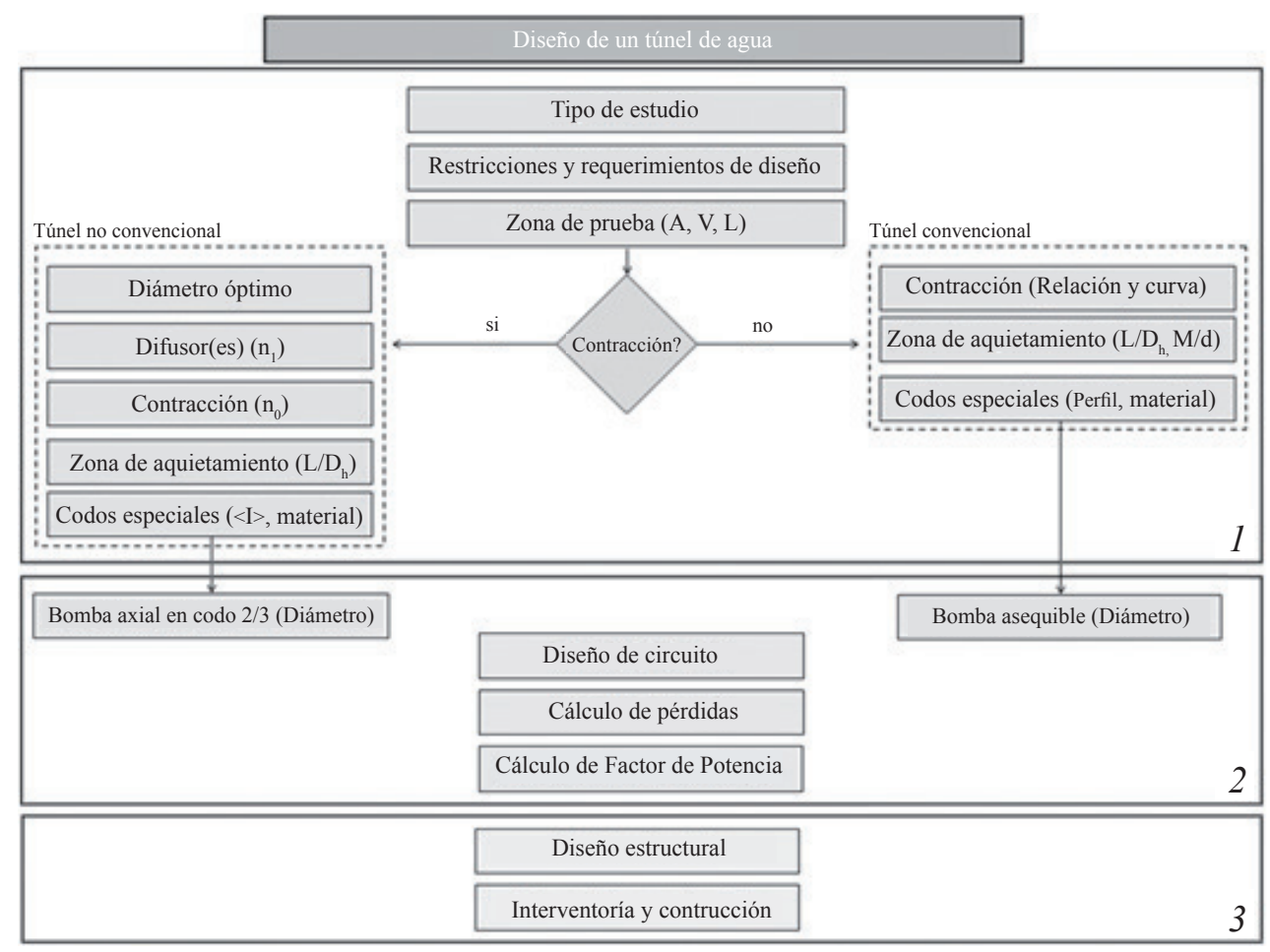

Figura 3. Metodología propuesta para el diseño de túneles de agua de mediana capacidad para investigación. Con: A-Área transversal, V-Velocidad del fluido, L-Longitud, Dh-Diámetro hidráulico de la celda, M-Tamaño de malla, d-Diámetro del alambre, no y n1-Relación de áreas de divergencia o convergencia, $\Phi$-Diámetro de tubería.

pruebas donde estas condiciones son diferentes (Lin, et al., 2006) (Smith, Sabatino y Praisner, 2001). Se espera que en estas condiciones se pueda investigar la interacción fluidoestructura en cargas cíclicas, vibraciones, fuerzas hidrodinámicas, potencia generada o consumida por máquinas y motores, respectivamente.

\subsubsection{Restricciones y requerimientos de diseño:} Las características de la Zona de prueba en este caso son: Rango de velocidades de $0-1.5 \mathrm{~m} / \mathrm{s}$ (según investigación sobre pruebas en equipos similares), Relación de áreas máxima entre modelo y Zona de prueba de $11.125 \%$ (Bernitsas, 2012), Diámetro de diseño para MH y PS de 0.45 $\mathrm{m}$ (investigación sobre pruebas similares; área transversal igual a $1.43 \mathrm{~m} 2$ ), Longitud de $5.60 \mathrm{~m}$ (para MH y PS se realizan mediciones hasta 10 diámetros y $5 \mathrm{~m}$ aguas abajo, respectivamente).

4.1.3 Normativa consultada. Requerimientos en: Diseño hidrodinámico, comportamiento y efectos del flujo a través de los componentes principales descrito en Ibarra (2010); Diseño experimental para MH (EquiMar Project Team, 2010) (Myers, 2012); Diseño estructural, cálculo de recipientes a presión descrito en ASME (2007), Starczewski (1981) y Annaratone (2007); ahorro energético en sistemas de bombeo, recomendaciones en la selección y operación de sistemas de bombeo como en Jones et al (2008), United States Department of Energy \& Hydraulic Institute (2006) y Nesbitt (2006).

\subsubsection{Acerca de la selección del tipo de túnel.} La Figura 2 muestra los tres tipos de túneles de agua. El uso de una contracción hace muy grande el tamaño de los conductos, a pesar de que en el mundo existen muchas instalaciones de este tipo como en Park et al (2003). Si los modelos bajo prueba son grandes o se requiere una Zona de prueba de gran sección transversal, es posible pensar en una instalación sin contracción (Figura 2c). No obstante, la opción más práctica 
especialmente para países como Colombia, es una instalación con difusor previo como la mostrada en la Figura $1 \mathrm{~b}$ debido a su sencillez y funcionalidad.

4.1.5 Diámetro óptimo. Existe un método en el cual se deriva la ecuación que relaciona la suma de las curvas de costos fijos (pérdidas de energía) y los costos de operación (sistema de bombeo) en función del Diámetro del conducto. La expresión desarrollada en Genić et al (2012) para un caudal fijo es:

$$
D_{r p f}^{5+m+x}=\frac{8(5+m) M}{\pi^{2}} \frac{(1+J) \cdot Y \cdot C_{a}}{X \cdot x \cdot E(1+F)(a+b)} \varepsilon^{m} \frac{G^{3}}{\rho^{2}}
$$

Dónde: rpf, subíndice para Rough Pipe Flow; $\mathrm{m}, \mathrm{M}, \mathrm{x}, \mathrm{X}$, parámetros del método; E, eficiencia combinada de motor y bomba; $\mathrm{F}$, factor que incluye el costo de válvulas, accesorios y su instalación; $\mathrm{a}$, amortización; $\mathrm{b}$, fracción que relaciona costos de mantenimiento anual; Y, horas de operación al año [horas/año]; Cen, costo de la Energía [USD/ Wh]; $\varepsilon$, rugosidad absoluta de la tubería [m]; G, flujo másico $[\mathrm{Kg} / \mathrm{s}]$; $\rho$, densidad del fluido $[\mathrm{Kg} /$ $\mathrm{m} 3$.

En este caso, para considerar el rango de velocidad de diseño $(0-1.5 \mathrm{~m} / \mathrm{s})$, se propone promediar los diámetros óptimos correspondientes a los valores extremos del rango, obteniendo $0.68 \mathrm{~m}$; según las recomendaciones de Nesbitt (2006), se selecciona el diámetro comercial mayor de $0.762 \mathrm{~m}$ (30 pulgadas). También, se puede basar en cálculo en la velocidad más importante del experimento.

\subsubsection{Cambios de sección: Difusor y contracción.}

Para acoplar la Zona de prueba con la tubería, fueron dimensionados cuatro elementos en sus extremos, que se muestran en la Figura 4. En la parte previa a la Zona de prueba, una transición divergente circular-rectangular (relación de áreas $\mathrm{n} 1=1.57$, longitud $=1.375 \mathrm{~m}$, ángulo de 8 grados) y un difusor tridimensional con placas internas como mecanismo de control de flujo (relación de áreas $\mathrm{n} 1=2.0$, longitud $=1.14 \mathrm{~m}$, ángulos totales de 30 y 24 grados - internos de 10 y 8 grados). En la parte posterior, una contracción con paredes curvas (relación de área no $=0.3189$ con relación $\mathrm{R} / \mathrm{Dh}=2$ ) y un cambio de sección rectangularcircular (no=1.0, longitud $1 \mathrm{~m}$ ). Datos tomados de Fried \& Idelchik (1989).

4.1.7 Codos especiales. Para controlar el flujo secundario propio de los cambios de dirección, normalmente se utilizan álabes especiales cuya construcción es dispendiosa y requiere de una gran precisión como lo menciona Pankhurst (1951). No obstante, para los mismos fines se propone el uso de accesorios especiales ya disponibles en el mercado nacional mediante importación,
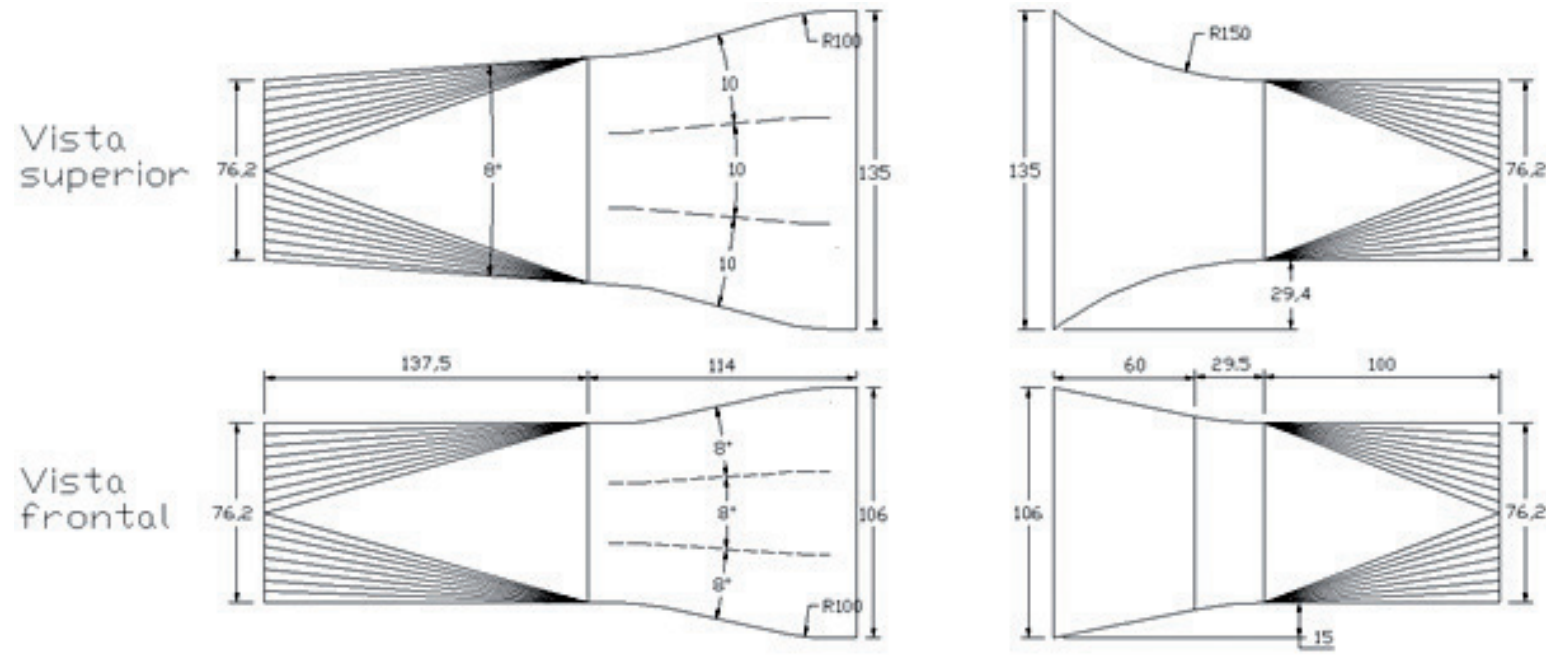

Figura 4. Elementos de la Zona principal del túnel (la Zona de prueba se instala en medio de ambos), medidas en cm. 


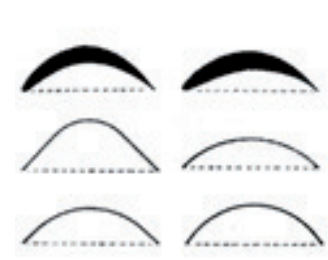

(a)

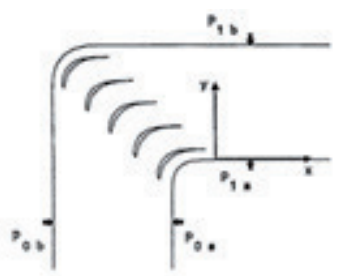

(b)

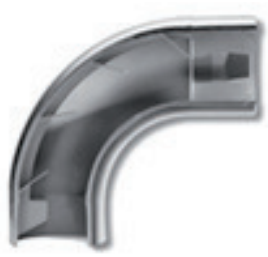

(c)

Figura 5. Elementos utilizados como codos o cambios de dirección en mecánica de fluidos. (a) Diferentes perfiles y (b) un ejemplo de su instalación en un túnel [29], (c) accesorio propuesto disponible en el mercado (corte meridional) [25].

prueba se instala en medio de ambos), medidas en $\mathrm{cm}$.

recomendados por United States Department of Energy \& Hydraulic Institute (2006), para uso en cualquier sistema de tuberías. Ofrecen ventajas hidrodinámicas como la baja pérdida de presión y la generación de un perfil de velocidades uniforme a la salida, lo cual también favorece las condiciones metrológicas de diferentes sensores industriales, Figura 5.

4.1.8 Simulación y validación: La validación de esta propuesta se realizó con Dinámica
Computacional de Fluidos utilizando el concepto del Laboratorio Virtual Simplificado presentado en Oslinger (2006), lo cual se puede ver en la Figura 6. El Software utilizado es ANSYS CFX V12.1, la convergencia de los modelos bidimensionales fue de 1x10e-4 en los valores RMS, con mallas estructuradas de hasta 111896 nodos. El modelo de turbulencia utilizado fue el k- $\varepsilon$. La validez de la velocidad uniforme como condición de frontera a la entrada la da el codo de diseño especial. En la entrada a la Zona de Prueba se evidencian

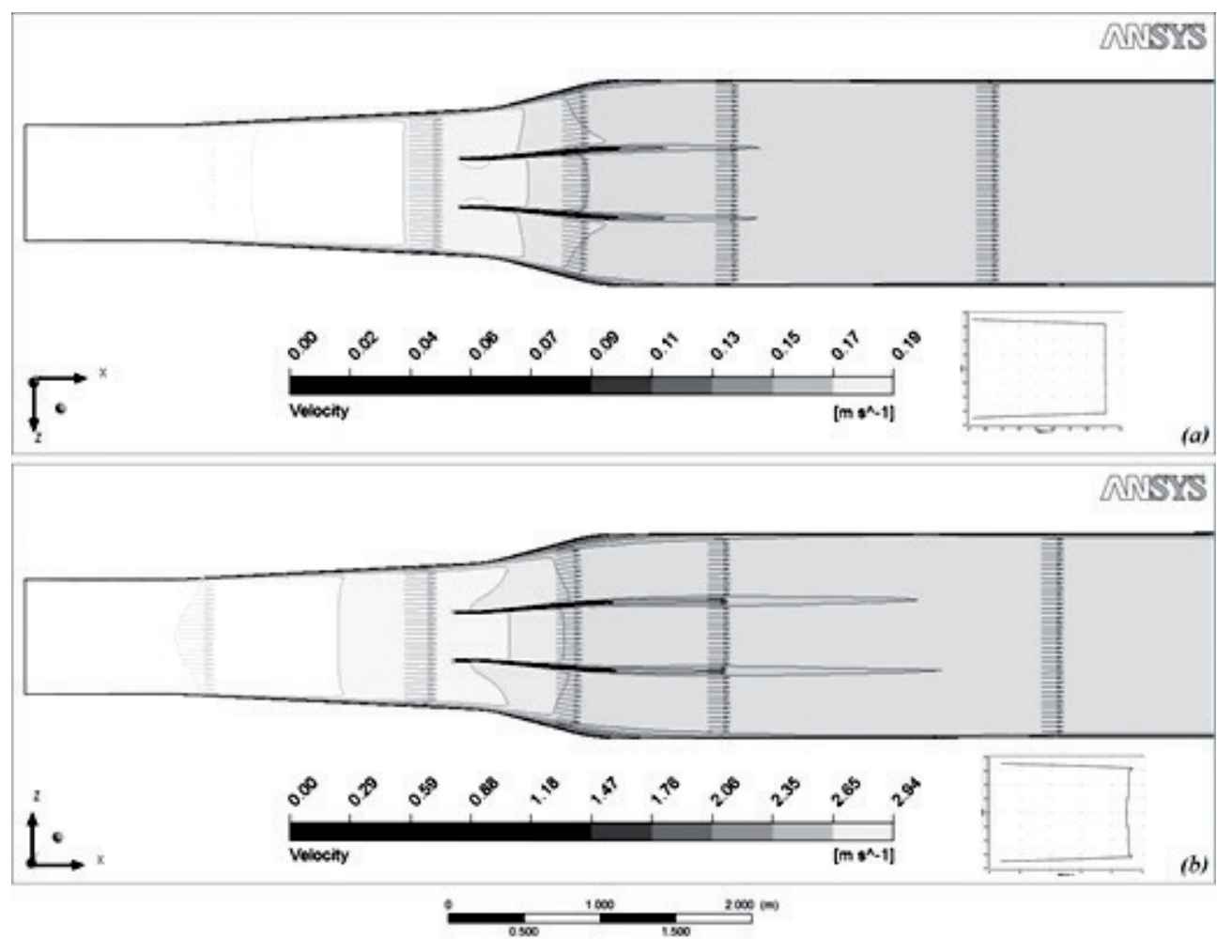

Figura 6. Campo y perfiles de velocidad según CFD para la geometría propuesta de la Zona principal del túnel, con velocidades de (a) $0.1 \mathrm{~m} / \mathrm{s}$ y (b) $1.5 \mathrm{~m} / \mathrm{s}$. 
posibles zonas de recirculación en las paredes exteriores de los difusores internos, con excepción del central. Para velocidades de 0.1 y $1.5 \mathrm{~m} / \mathrm{s}$ en la zona de prueba es posible conseguir un perfil de velocidades favorable con menos del $5 \%$ de intensidad de turbulencia a distancias de 0.95 $\mathrm{m}(4.50 \%)$ y $1.75 \mathrm{~m}(4.93 \%)$ desde el borde de salida de las placas de división, respectivamente, como en plataformas similares (Germain, 2006). Para no restar longitud útil a la Zona de prueba, se ha calculado que con una pantalla de celdas hexagonales con un diámetro hidráulico de 0.067 $\mathrm{m}$ y una longitud de $0.7 \mathrm{~cm}$, con lo que según Germain et al (2005), se obtendría intensidades del $5 \%$ en $0.3 \mathrm{~m}$ aproximadamente.

\subsection{Sistema de bombeo y sistema de tuberías}

A pesar de que las bombas de tipo axial ubicadas en un codo son las más utilizadas en estas instalaciones (Figura 1), la investigación realizada pudo evidenciar que para el tipo de túnel dimensionado también es posible instalar cualquier bomba hidráulica centrífuga y de flujo mixto que pueda cumplir, por supuesto, con los requerimientos de caudal y carga (o presión). Esto resulta porque el diámetro hidráulico del conducto para este tipo de túneles es menor que para uno convencional, aumentando la carga necesaria a este tipo de bombas según el caudal necesario, que puede dar una idea inicial sobre el número y tipo de bombas requeridas. La mayor ventaja es que se utiliza una menor cantidad de agua.

\subsubsection{Consideraciones sobre diseño y selección.}

En este punto del diseño solo se conoce el caudal requerido, por lo que hay que realizar algunas consideraciones para el sistema de bombeo. En primer lugar, se determina que el circuito sea cerrado para que la carga dinámica sea la única para la bomba y poder aplicar estrategias de ahorro en bombeo, lo que no se puede hacer con la carga estática. Por su parte, la bomba hidráulica puede provenir de modelos disponibles o ser adquirida con la obligatoria asesoría por parte de distribuidores en el país (Figura 7). La recomendación es utilizar hasta dos bombas en paralelo y en el caso de las centrifugas, respetar la zona de operación para evitar problemas de cavitación, vibración y sobrecalentamiento como lo recomienda Forsthoffer (2011). La instalación de más de una bomba agregará perdidas en el circuito debido a los accesorios que deban ser adicionados al circuito, con su correspondiente efecto sobre la potencia consumida.
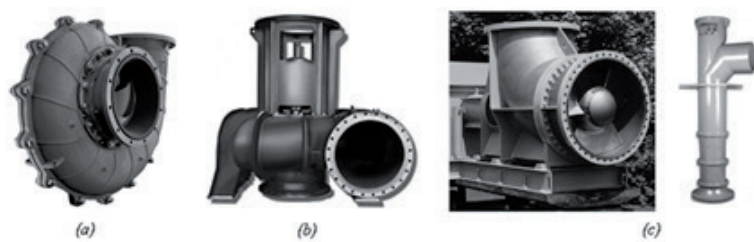

Figura 7. Bombas hidráulicas de marcas con distribuidores en el país. (a) Bomba centrifuga, (b) bomba de flujo mixto y (c) bomba axial para instalación en codo y de pozo.

Posteriormente, se realiza un diseño iterativo de las tuberías y accesorios necesarios para conectar la zona principal y la bomba hidráulica (Fried \& Idelchik, 1989), seguido del cálculo de pérdidas finales y de los parámetros de la bomba; proceso que termina cuando se conoce si la bomba seleccionada es la indicada ( $\sin$ sobredimensionamiento). Para seguir con los pasos de la metodología, se selecciona una bomba axial instalada en codo. Esto obedece al hecho de que permiten doble regulación en la velocidad del fluido con la regulación de la orientación de sus álabes tipo Kaplan y la velocidad de rotación, además, algunos modelos tienen elementos para direccional el flujo para una menor pérdida de presión en el codo, lo que disminuye la magnitud de las pérdidas totales de energía y la potencia de bombeo aproximadamente igual a $60 \mathrm{HP}$ (asumiendo una eficiencia del $85 \%$ en la bomba).

4.2.2 Factor de Potencia. El Factor de potencia $(\lambda)$ de la instalación es una medida del ahorro energético. Según Gleed \& Saiva (1967), está expresado como la relación entre la energía por unidad de tiempo disponible en la zona de prueba y la potencia de bombeo, cuya magnitud se considera óptima en la medida en que esté más cercana a cero. En nuestro caso, $\lambda$ es igual a 0.07 . 


\subsection{Cálculo estructural}

4.3.1 Zona de prueba. El espesor del vidrio de observación se determina considerando la teoría de placas (Starczewski, 1981). El espesor necesario en este caso es de 18 milímetros para una distancia entre apoyos de $0.65 \mathrm{~m}$, considerando un factor de seguridad de 4 para vidrio templado (Ibarra, 2010).

\subsubsection{Conductos: Tubería y secciones} rectangulares. Seleccionando acero al carbono pensando en un la ventaja de resistencia y disminución de costos. No obstante, debe ser complementada con la aplicación de una capa de resina epóxica sobre todas las superficies internas, esta tendencia actual se conoce como epoxycoated pipelines. Los revestimientos también se encuentran disponibles en el mercado nacional. Las tuberías calculadas a rigidez, tienen un espesor de 15.88 milímetros para deformaciones por debajo del $1 \%$ considerando el caso extremo de estar soportadas sólo en sus extremos (Annaratone, 2007). A su vez, las secciones rectangulares pueden ser construidas con placas de acero de 6.35 milímetros $\mathrm{m}$ de espesor (The American Society of Mechanical Engineers,
2007). La instalación propuesta puede verse en la Figura 8.

\section{Conclusiones}

Los túneles de agua son instalaciones de pruebas experimentales muy utilizadas en todo el mundo debido a su importancia en la investigación. Este trabajo muestra que pueden implementarse en el país si se comprenden realmente su utilidad y los conceptos relacionados con su diseño. Según la investigación p-2010-1512 apoyada por COLCIENCIAS, las instalaciones de mediano y gran tamaño pueden ser viables a corto o mediano plazo si son concebidas con ayuda de la la metodología presentada y desarrollada en forma inicial en este artículo, debido a la disponibilidad de casi todos los elementos en el mercado nacional, incluyendo los variadores de velocidad para los motores eléctricos de bombas pero no en el caso de las pantallas de celdas hexagonales.

La zona de prueba del túnel es única según los requerimientos de los experimentos, sin embargo, pueden acoplarse a varios sistemas de bombeo (tuberías, accesorios, equipos) según el tipo de bomba hidráulica e instrumentación disponible.

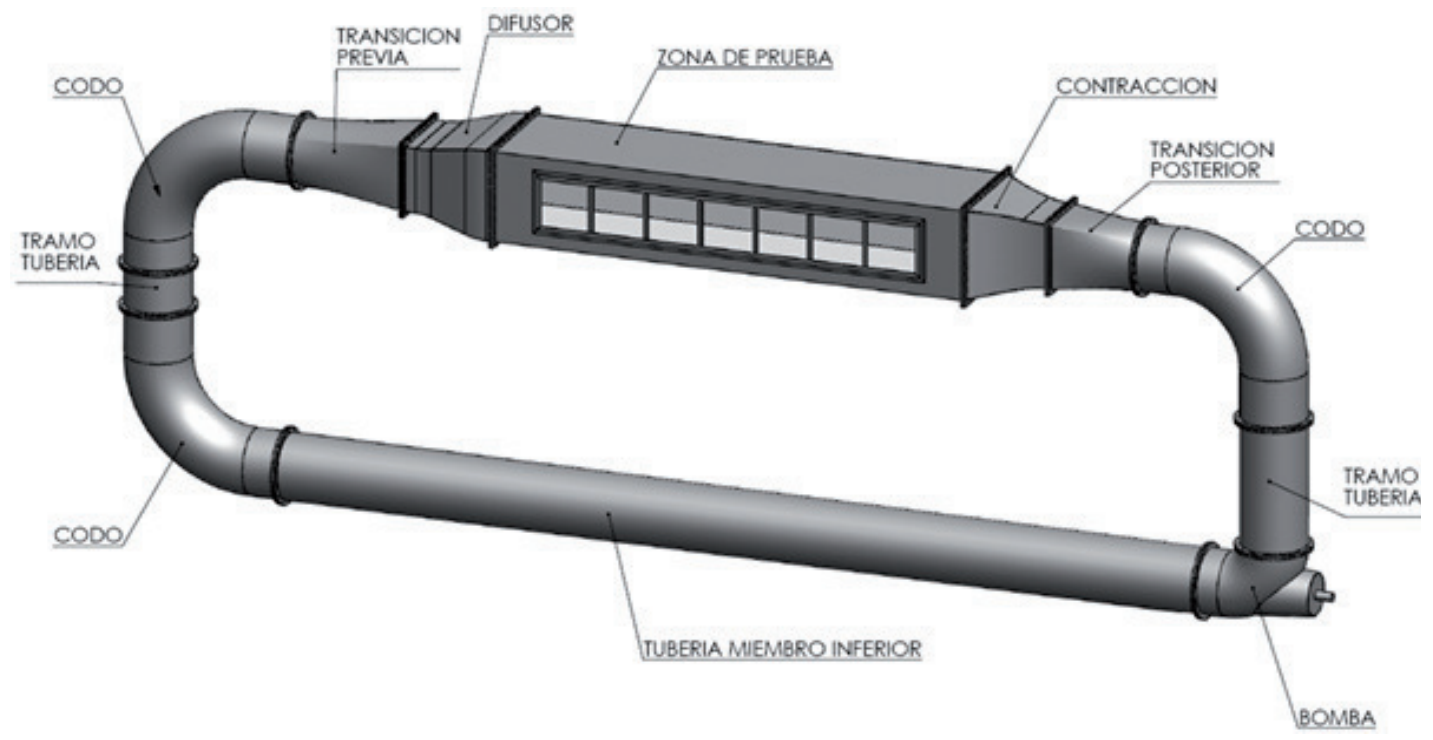

Figura 8. Instalación dimensionada para la evaluación de Máquinas Hidrocinéticas y Pequeños Submarinos para exploración de potencial energético. 
Se recomienda aplicar estrategias de ahorro energético en sistemas de bombeo como el uso de variadores electrónicos de velocidad y elementos de control de flujo como los cambios de dirección (codos) especiales para evitar la generación de pérdidas excesivas de presión como se sugiere Pankhurst. Es importante que en una propuesta de diseño se tenga en cuenta, si así se requiere, el uso de sistemas intercambiadores de calor, de limpieza y filtración del agua, control del aire disuelto, entre otros, para garantizar la continuidad operativa de las pruebas sin descuidar la magnitud del factor de potencia; algunos valores están en Gleed \& Saiva (1967).

\section{Agradecimientos}

A COLCIENCIAS por el apoyo a esta investigación como parte del Programa Jóvenes Investigadores e Innovadores "Virginia Gutiérrez de Pineda". De igual forma, se reconoce toda la oportuna y amable comunicación e intercambio de ideas con los siguientes profesores:

- Michael M. Bernitsas, PhD. University of Michigan, EEUU. (michaelb@umich.edu)

- Luke Myers Ph.D. University of Southampton, UK. (luke@soton.ac.uk)

- Bo Yang, Ph.D. Shanghai Jiao Tong University. (byang0626@sjtu.edu.cn)

\section{Referencias bibliográficas}

Annaratone, D. (2007). Pressure Vessel Design. Milan: Springer.

ASME - The American Society of Mechanical Engineers, 2011. A Closed Circuit Closed Jet, High-speed, Water Tunnel: Gardfield Thomas Water Tunnel. http://files.asme.org/ASMEORG/ Com munities/Hist ory/ Landmarks/5552.pdf.

ASME - The American Society of Mechanical Engineers (2007). Sección VIII, Division 1, Mandatory App. No.13: Rules for construction of Pressure vessels. ASME: Estados Unidos.
Bahaj, A., Batten, W. \& McCann, G. (2007). Experimental verifications of numerical predictions for the hydrodynamic performance of horizontal axis marine current turbines. Renewable Energy (32) 15, 2479-2490.

Bernitsas, M., michaelb@umich.edu, 2012. Request for information. [email] Mensaje para G.A. Ibarra (german.ibarra@correounivalle.edu. co). Enviado 23 Febrero 2012, 13:30.

Cummings, R. \& Morton, S. (2006). Continuing evolution of aerodynamic concept development using collaborative numerical and experimental evaluations. Proceedings of the Institution of Mechanical Engineers, Part G: Journal of Aerospace Engineering 220 (6), 545-557.

EquiMar Project Team, 2010. EquiMar Project Deliverable 3.4: Best practice for tank testing of small marine energy devices. http://www.equimar. org/equimar-project-deliverables.html.

FML - Functional Morphology Laboratory at University of Rhode Island, 2012. Suction feeding in spiny dogfish. http://www.uri.edu/cels/ bio/wilga/Personnel.html

Forsthoffer, W. (2011). Forsthoffer's Best practice Handbook for Rotating Machinery. Langford Lane: Butterworth-Heinemann.

Fried, E. \& Idelchik, I.E. (1989). Flow Resistance: A Design Guide for Engineers. United States: Taylor \& Francis Group.

Genić, S., Jaćimović, B. \& Genić, V. (2012). Economic optimization of pipe diameter for complete turbulence. Energy and Buildings (45), 335-338.

Germain, G., 2006. Experimental Facilities. Hydrodynamics Facilities. IFREMER Newsletter 1-6 Oct. http://wwz.ifremer.fr/manchemerdunord/ content/download/26767/374089/file/IHF_ NewsLett er_Issue1.pdf

Germain, G., Candelier, C. \& Blarel, S. (2005). Structures alvéolaires et homogénéisation d'écoulement. In Proceedings of the 10èmes 
Journées de 1'hydrodynamique, Nantes, France, p. 1-10.

Gleed, D. \& Saiva, G. (1967). A Selective survey of literature on water tunnels to provide a basis for the design of the Lucas Heights Water Tunnel. Technical Report OSTI 4537313 for the Australian Nuclear Science Technology Organisation. Australia.

Ibarra, G. (2010). Diseño de un túnel de agua. Trabajo de grado, Escuela de Ingeniería Mecánica, Universidad del Valle, Cali, Colombia.

Jones, G., Sanks, R., Tchobanoglous, G. \& Bosserman II, B. eds. (2008). Pumping station Design. Montana: Butterworth \& Heinemann.

Klemm, T. \& Gabi, M. (2003). Using PIV and $C F D$ to investigate the effect of casing design on cross flow fan performance. In Proceedings of PSFVIP-4, Chamonix, France, p. 1-8.

LHM - Laboratory for Hydraulic Machines, 2011. Research: Cavitation High Speed Visualization. http://lmh.epfl.ch

Lin, Y., Brant, D., Bartlett, R., Hirschl, R. \& Bull, J. (2006). Pulsatile Flow past a Cylinder: An Experimental Model of Flow in an Artificial Lung. ASAIO Journal 52 (6), 614-623.

Lienhard, J. IV y Lienhard, J. V. (2003) A heat transfer textbook. Cambridge: Phlogiston Press.

Myers, L., 1.e.myers@soton.ac.uk, 2012. Request for information. [email] Mensaje para G.A. Ibarra (german.ibarra@correounivalle.edu.co). Enviado 16 Febrero 2012, 11:07.

Nesbitt, B. (2006). Handbook of pumps and pumping: Pumping manual international. Oxford: Elsevier Science Ltd.

Oslinger, J., Palacios, J. \& Jaramillo, G. (2005). Simplified Virtual Laboratory's Experience on Multiphysics Modeling. In Procedings of VIII International Conference on Computational Plasticity - Fundamentals and Applications COMPLAS, Barcelona, Spain, p. 1071-1074.
Pankhurst, R.C. (1951). Wind-Tunnel Technique. London: Sir Isaac Pitman \& Sons, Ltd.

Park, J., Cutbirth, J. \& Brewer, W. (2003). Hydrodynamic performance of the large cavitation channel (LCC). In Proceedings of FEDSM'03 4th ASME-JSME Joint Fluids Engineering Conference, Honolulu, EEUU, p.1-14.

Ripken, J. (1951). Design studies for a ClosedJet Water tunnel. Technical Paper No.9, Series B. University of Minnesota, St. Anthony Falls Hydraulic Laboratory. Minneapolis, Unites States.

Shaughnessy, E., Katz, I. y Schaffer, J. (2005) Introduction to Fluid Mechanics. New York: Oxford University Press, Inc.

Smith, C., Sabatino, D. \& Praisner, T. (2001). Temperature sensing with thermochromic liquid crystals. Experiments in Fluids (30) 2, 190-201.

Starczewski, M. (1981). Non-Circular pressure vessels: Some guidance notes for designers. British Engine Technical Report, Volume XIV.

Subramanian, C., Shinjo, N. \& Gangadharan, S. (2004). A Study of hydrodynamic characteristics of Boundary Layer with Algae roughness. Marine Technology 41 (2), 60-65.

United States Department of Energy \& Hydraulic Institute. (2006). Improving Pumping System Performance: A sourcebook for Industry. Golden (Colorado), National Renewable Energy Lab.

VHE - Vortex Hydro Energy, 2012. VIVACE Tech. www.vortexhydroenergy.com.

Yang, B., byang0626@sjtu.edu.cn, 2012. Request for information. [email] Mensaje para G.A. Ibarra (german.ibarra@correounivalle.edu.co). Enviado 12 Abril 2012, 21:10.

Warzinski, R., Lynn, R., Robertson, A. \& Haljasmaa, V. (2000). Development of a highpressure water tunnel facility for ocean $\mathrm{CO} 2$ storage experimentation. Division of Fuel Chemistry American Chemical Society 45 (4), 809-813. 